\title{
Radical-mediated Dimerization and Oxidation Reactions for the Synthesis of Complex Alkaloids
}

\author{
Stephen P. Lathrop, Justin Kim, and Mohammad Movassaghi
}

\begin{abstract}
Concise and general strategies for radical-based dimerization of cyclotryptamine and cyclotryptophan derivatives in addition to radical-based oxidation of structurally complex diketopiperazines are discussed. The impact of these radical-based chemistries on the rapid generation of molecular complexity is highlighted using representative examples from recent complex homodimeric alkaloid total syntheses. Additionally, a new and general strategy for radical-based directed heterodimerization of cyclotryptamine substructures via solventcaged unsymmetrical diazene fragmentation is discussed.
\end{abstract}

Keywords: Alkaloid · Diazene fragmentation · Hexahydropyrroloindole · Radical dimerization ·

Radical oxidation

Radical chemistry has long been recognized as an important tool for the rapid generation of molecular complexity. Radical intermediates have proven their utility in numerous cyclization cascades, ${ }^{[1]}$ formation of challenging sterically congested bonds, ${ }^{[2]}$ and as key intermediates en route to the functionalization of ofteninert chemical motifs. ${ }^{[3]}$ Through our studies in the area of cyclotryptamine and cyclotryptophan-derived natural products, we have not only harnessed the power of radical chemistry to access natural products in a concise manner, but we have also highlighted how the orthogonal reactivity profile of radical intermediates can be used to efficiently elaborate intricate substructures that are highly sensitive to polar transformations. ${ }^{[4]}$ As seen in the following examples, radical chemistry is an invaluable tool in the chemist's quest to navigate the ever-increasing complexities as they seek to expand the frontiers of total synthesis.
Consistent with the hypotheses set forth in the 1950s by Woodward and Robinson, ${ }^{[5]}$ the biogenesis of the dimeric cyclotryptamines and the more complex dimeric epidithiodiketopiperazines is believed to arise from the oxidative radical dimerization of tryptamine and tryptophan-derived monomeric subunits. This dimerization mode effectively establishes the vicinal quaternary carbon centers in a highly convergent manner. While the viability of an oxidative dimerization route had previously been demonstrated for the syntheses of carbon-carbon linked dimeric alkaloids such as (+)-chimonanthine (3, Scheme 1), each of these methods produced a mixture of $\mathrm{C}(2)$-symmetric and meso $\mathrm{C}(3)-\mathrm{C}\left(3^{\prime}\right)$ dimeric cores. ${ }^{[6]}$ In contrast, we sought a practical strategy to address the issue of both relative and absolute stereochemical control in the synthesis of such a prevalent core structure. ${ }^{[7]}$ Seeking to exploit the convergent nature and efficiency of a radical-radical recombination event, we opted to access the radical species through a stepwise $2 \mathrm{e}^{-}$oxidation followed by $1 \mathrm{e}^{-}$reduction. Our first application of this approach is highlighted in our enantioselective synthesis of (+)-chimonanthine (3, Scheme 1). ${ }^{[8]}$ Encouraged by seminal reports by Crich and Danishefsky[9] on the utility of $\mathrm{C}(3)$-functional cyclotryptamine derivatives, ${ }^{[10]}$ we envisioned the tricyclic benzylic bromide (+)-1 and related derivatives ${ }^{[1]}$ to serve as versatile precursors to the desired radical intermediates en route to homodimeric complex alkaloids. The cisfused 5,5-bicyclic ring system preserves the stereochemistry at the tertiary benzylic stereocenters even despite reduction of the benzylic halide to a stereochemically labile radical species.

After significant experimentation, we recognized that the desired single electron reduction of the tertiary benzylic bromide (+)-1 could be most practically accomplished using tris(triphenylphosphine) cobalt(I) chloride. ${ }^{[8]}$ The rapidity of this reduction generates a large concentration of radicals, which undergo radical recombination and afford the desired dimeric advanced intermediates of interest. Treating intermediate $(+)-\mathbf{1}$ with this cobalt complex yielded dimer (+)-2 in $60 \%$ isolated yield on multi-gram scale, affording a concise synthetic solution to (+)-chimonanthine (3) and other related calycanthaceous alkaloids. ${ }^{[8]}$

Notably, our Co(I)-promoted radical dimerization of cyclotryptamine derivatives has been successfully applied to the synthesis of a number of other alkaloids..$^{[4,11,12]}$ In particular, this chemistry enabled the first total synthesis of (+)-11,11'-dideoxy-

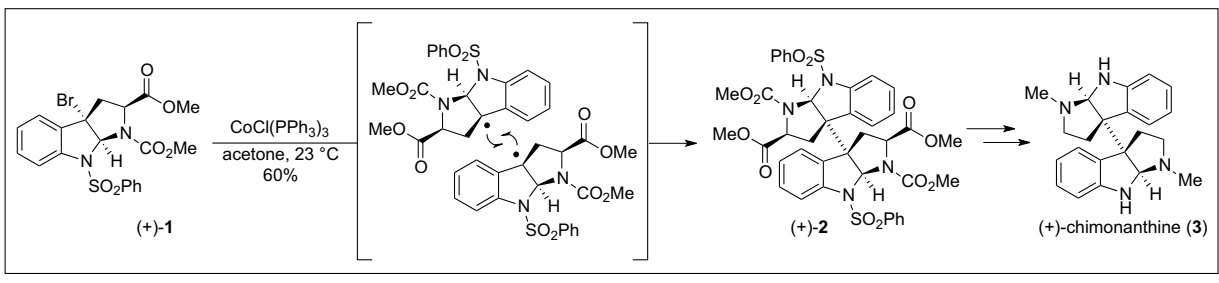

Scheme 1. 
verticillin A (9, Scheme 2) which embodied the intricate and fascinating molecular architecture of dimeric epidithiodiketopiperazine alkaloids. ${ }^{[4]}$ Treatment of tetracyclic bromide (+)-4 with this cobalt complex afforded the dimer (+)-5 in $46 \%$ yield en route to $(+)-9$ (Scheme 2$)$. With rapid access to the dimeric molecular framework in only six steps from commercially available material, we sought the development of a general strategy to access the more highly functionalized epidithiodiketopiperazine natural products. We had postulated ear$1 y^{[13]}$ in our retrobiosynthetic analysis ${ }^{[14]}$ that the existing carbon-sulfur bonds in the natural product may arise from the incorporation of a nucleophilic sulfur source via intermediacy of an $\mathrm{N}$-acyliminium ion. ${ }^{[15]}$ We envisioned access to such a reactive intermediate by ionization of a hemiaminal that in turn could be prepared by hydroxylation of the $\mathrm{C}_{\alpha}$ centers of the diketopiperazine. ${ }^{[16]}$

Our initial attempts to oxidize this amino acid residue via enolate chemistry were fraught with complications arising from the formation of complex diastereomeric mixtures, partial hydroxylation, as well as decomposition products. We quickly discovered that the hemiaminal substructure found in the desired product (+)-6 was extremely labile to both acidic and basic conditions, rendering such conditions incompatible with the desired transformation. We thus turned our attention to methods based on radical chemistry. We expected the $\mathrm{C}_{\alpha}-\mathrm{H}$ bond of the amino acid residues to be particularly labile toward radical abstraction owing to the captodative stabilization afforded by the adjacent carbonyl and amide functional groups. ${ }^{[4]}$ While we found that $\mathrm{C}_{\alpha}-\mathrm{H}$ abstraction was unfeasible using carbon- or oxygen-based radical sources, with the former resulting in no reaction and the latter resulting in substrate decomposition, after significant experimentation, we discovered that bromine radical sources were able to oxidize the $\mathrm{C}_{\alpha}$-centers of simple monomeric substrates efficiently. We reported that the use of $\mathrm{N}$-bromosuccinimide and AIBN as an initiator in refluxing carbon tetrachloride afforded us the oxidized product (Scheme 3). ${ }^{4]}$ The $\mathrm{C}_{\alpha}-\mathrm{Br}$ bond, however, was extremely labile in the absence of proximal inductively withdrawing substituents, resulting in rapid ionization and elimination to the enamide substructure, which subsequently underwent further bromination to a tetrabrominated intermediate 11. ${ }^{[17]}$ Reduction of this compound with sodium iodide then afforded a bis $(\alpha, \beta$-unsaturated $)$ diketopiperazine 12 with the desired oxidation state. Notably, under acidic conditions, diene 12 was a competent precursor to the $N$-acyliminium ions required for the anticipated nucleophilic thiolation. ${ }^{[4]}$

We were, however, unable to translate this oxidation-thiolation approach to the more complex dimer owing to the additional sensitivity of the $\mathrm{C}(3)-\mathrm{C}\left(3^{\prime}\right)$ bond toward fragmentation during formation of an analogous tetraene intermediate. Careful analysis of the bond dissociation energies (BDE) involved in our earlier bromine-mediated radical abstraction combined with the realization that the average BDE of a formyl $\mathrm{C}-\mathrm{H}$ bond was approximately that of an $\mathrm{H}-\mathrm{Br}$ bond led us to explore the use of permanganate-based reagents to effect the desired tetrahydroxylation reaction. In organic solvents, several studies have demonstrated that aldehydes are oxidized by permanganate reagents to their corresponding carboxylic acids via a radical mechanism. ${ }^{[18]}$ Indeed, the use of tetrabutylammonium permanganate in pyridine, conditions originally developed

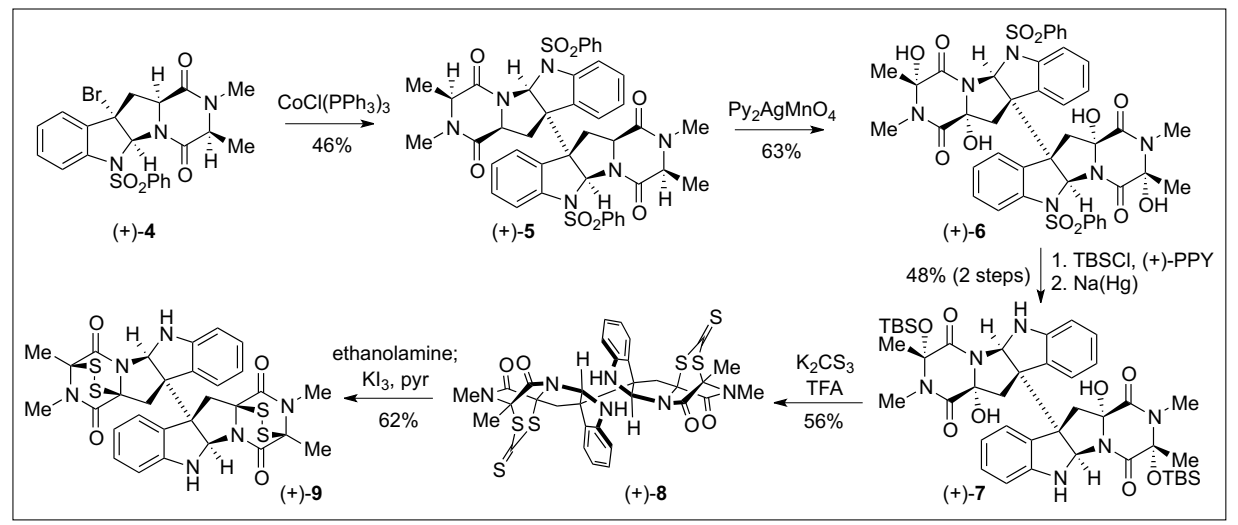

Scheme 2 . for aldehyde oxidation, ${ }^{[19]}$ on our dimeric substrate (+)-5 afforded the first samples of the desired tetrahydroxylated intermediate (+)-6 in 9\% yield. The yield was improved to $40 \%$ upon refinement of our isolation techniques for this highly polar and sensitive tetrahydroxylated compound. Ultimately, optimizing around solvent and counterion led us to employ $\mathrm{Py}_{2} \mathrm{AgMnO}_{4}$ in dichloromethane, which afforded the desired product in 63\% yield (Scheme 2). Importantly, the tetrahydroxylated product was obtained as a single diastereomer. It was revealed through X-ray diffraction analysis that all four hydroxylations had occurred with retention of stereochemistry, consistent with a rapid abstractionrebound mechanism. ${ }^{[20]} \mathrm{A}$ compilation of our studies employing a variety of conditions, including the successful application of tetrabutylammonium permanganate as described above, indicate that this is a permanganate-mediated rather than a silvermediated transformation. The oxidation of a cyclopropyl hydantoin radical probe 13 (Scheme 4) indicated that the rebound carbon-oxygen bond formation occurs on a faster time-scale than the ring opening of the expected cyclopropyl methyl radical intermediate. ${ }^{[4]}$ With gram-scale access to our key tetraol intermediate, we were able to evaluate a variety of sulfidation methods, the most successful of which involved the acid-mediated ionization of the hemiaminals and trapping with a trithiocarbonate. Rapid unraveling of the bis(dithiepanethione) using ethanolamine in acetone followed by an oxidative workup resulted in the first total synthesis of a dimeric epidithiodiketopiperazine, namely the natural alkaloid (+)-11,11'-dideoxyverticillin A (9, Scheme 2$)$.

Having secured a general method for the synthesis of the core molecular framework of the homodimeric cyclotryptamine and epidithiodiketopiperazine based alkaloids, we sought a general method for the synthesis of the corresponding heterodimeric molecular foundation. Such heterodimeric structures are found in a variety of natural products such as meso-chimonanthine ${ }^{[21]}$ and (+)-leptosin K. ${ }^{[22]}$

Although the previously described cobalt-mediated radical homodimerization of cyclotryptamine derived subunits has proven successful for the synthesis of a number of natural products, the application of this chemistry to the synthesis of heterodimeric substructures proved

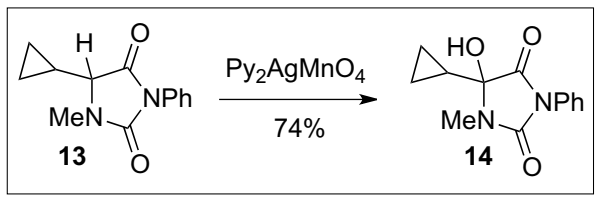

Scheme 4. 
challenging. For example, treatment of a racemic mixture of tricyclic bromide $( \pm)$ 15 with tris(triphenylphosphine)cobalt(I) chloride resulted in the formation of the desired meso-16 in only $16 \%$ yield along with ( \pm )-17 (16\% yield) (Scheme 5).[23] The unselective nature of this dimerization reaction in addition to the presence of undesired disproportionation products, and the complications in product isolation from such a complex mixture, restricts the use of this chemistry for advanced stage heterodimer formation. In order to circumvent these challenges, we recently developed a general strategy for the directed synthesis of heterodimeric cyclotryptamines. ${ }^{[23]}$ This process is based on the photolytic fragmentation of dialkyl diazenes to form localized radicals whose recombination within a solvent-cage resulted in the selective formation of the desired heterodimeric products.

Our synthetic strategy centered on the expulsion of dinitrogen from an unsymmetrical diazene $\mathbf{1 8}$ to form a pair of carbon-centered radicals whose recombination would result in the formation of the desired heterodimer 19 (Scheme 6). Despite the rich history of dialkyl diazenes in the field of physical organic chemistry, ${ }^{[24]}$ their utilization in complex molecule synthesis has been examined sparingly. Although ring contraction via the use of cyclic diazenes has precedence in complex molecule synthesis, ${ }^{[25]}$ the corresponding acyclic diazene derivatives have received far less attention possibly due to the additional challenge of intermolecular recombination of the resulting radical pair upon extrusion of dinitrogen. ${ }^{[26]}$ Indeed, disproportionation and crossover products are often observed as major byproducts in the context of acyclic dialkyl diazene fragmentation.

We first examined the practicality of our diazene based fragment assembly in the context of the homodimerization of complex cyclotryptophan derivatives (Scheme 7). The symmetrical diazene (+)-21 was prepared via oxidation of the corresponding sulfamide (+)-20 in the presence of $\mathrm{N}$-chlorosuccinimide and 2-tert-butylimino-2-diethylamino-1,3dimethylperhydro-1,3,2-diazaphosphorine (BEMP). We found that irradiation of (+)-21 led to expulsion of dinitrogen and formation of the desired dimeric hexacycle (+)-17 in $60 \%$ yield. The overall efficiency of the process was improved when the freshly prepared crude diazene was used without chromatographic purification. A representative synthesis of a mixed sulfamide is illustrated in Scheme 8. The sulfamoyl chloride 22, accessed from the corresponding amine (+)-19 (Scheme 7), was treated with amine $\mathbf{2 3}$ (Scheme 8 ) to afford the desired mixed sulfamide (+)-24 in $86 \%$ yield. Oxidation of the sulfamide

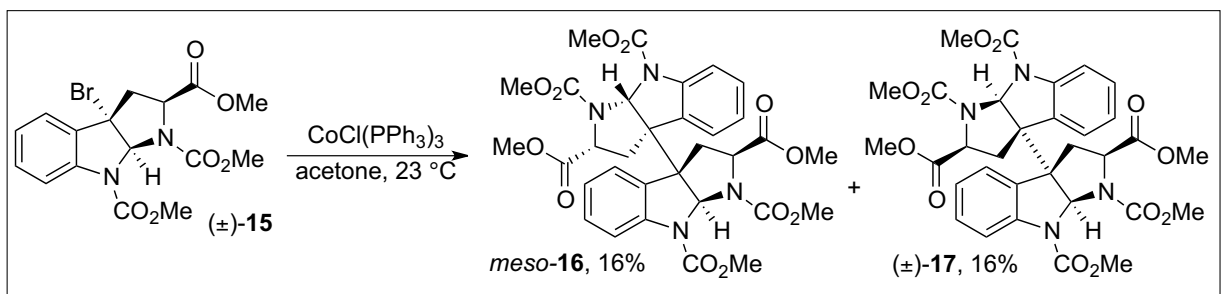

Scheme 5.

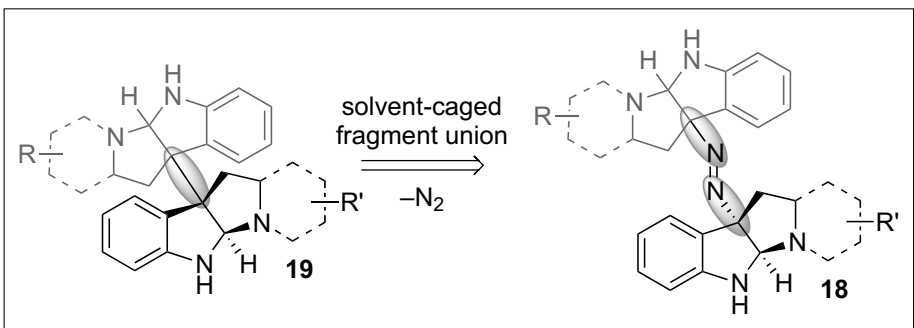

Scheme 6.

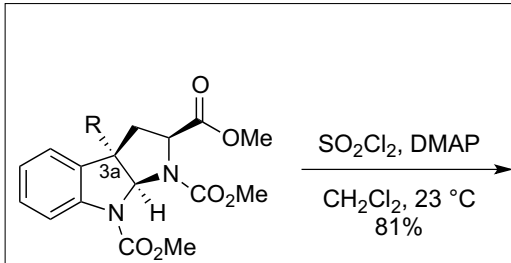
1. $\mathrm{TMSN}_{3}, \mathrm{SnCl}_{4}$
2. dithiothreitol
$71 \%$ (2-steps) $\stackrel{(+)-15, \mathrm{R}=\mathrm{Br}}{(+)-19, \mathrm{R}=\mathrm{NH}_{2}}$
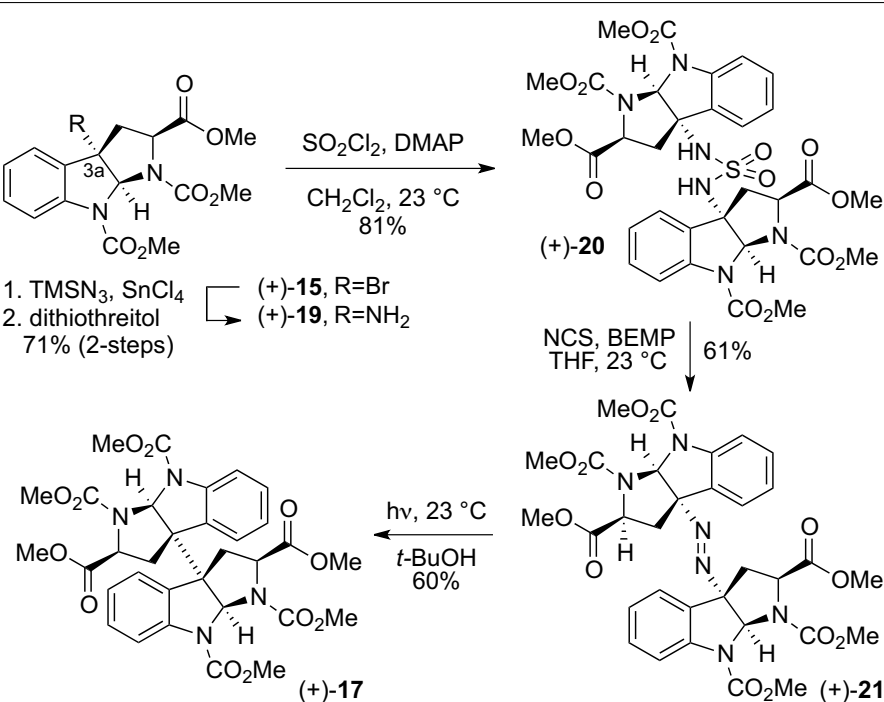

Scheme 7.

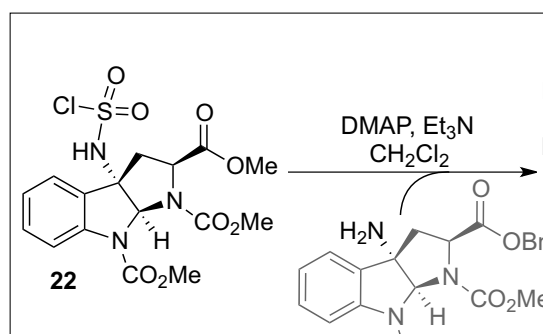

$23 \mathrm{CO}_{2} \mathrm{Me}$

$(+)-20$
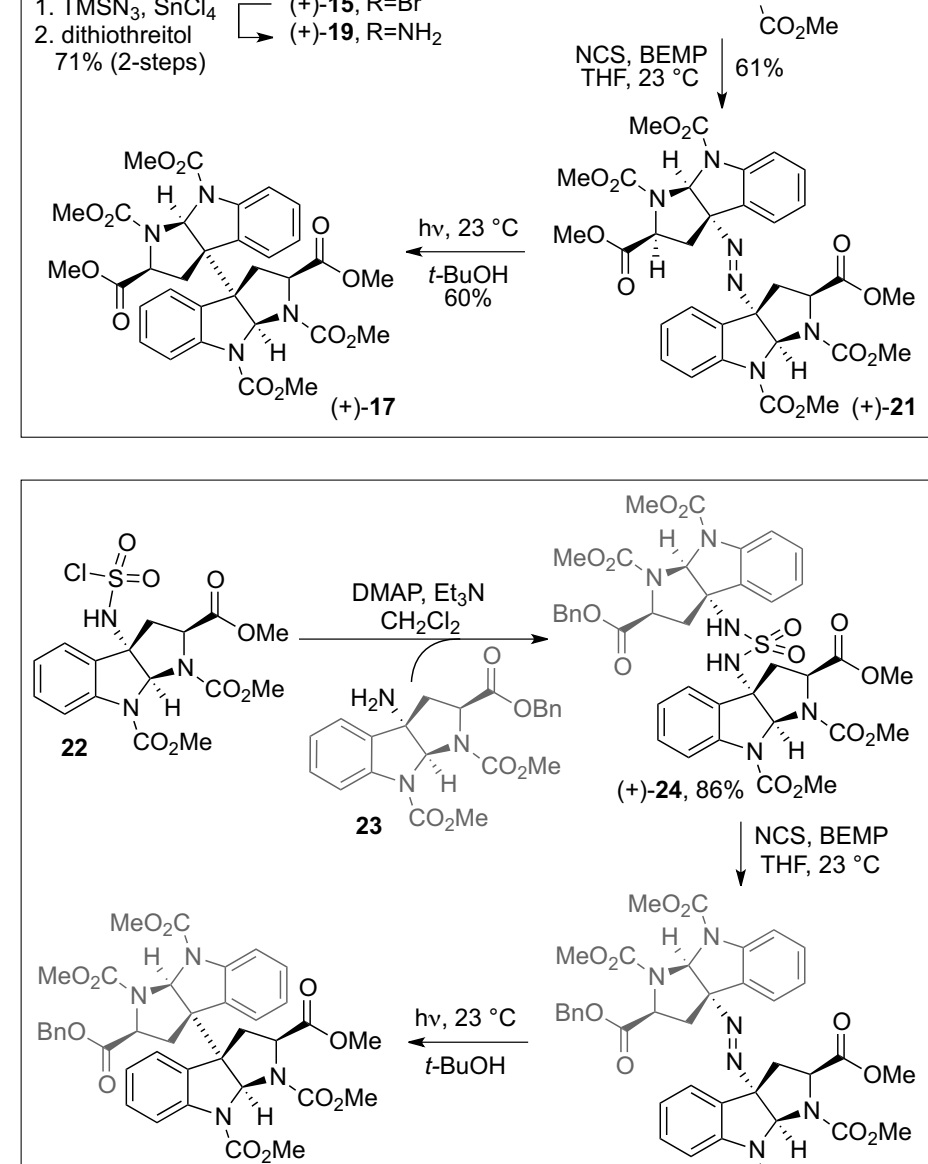

(+)-26 70\% (68\% 2 steps)

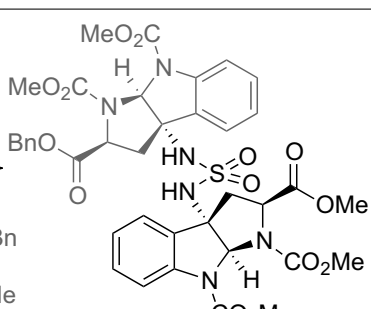

Scheme 8

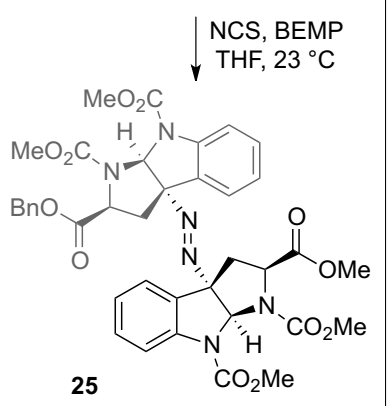

as described above provided the desired diazene 25 which upon irradiation in tertbutanol afforded the desired heterodimer (+)-26 in $68 \%$ yield from (+)-24. Notably, neither of the two possible homodimeric products was observed by HPLC analysis of the crude product mixture.

Further insight into the critical directed fragment assembly step in a solvent-cage was gleaned from exposure of an equal mixture of symmetrical sulfamides $\mathbf{2 0}$ and 27 (Scheme 9) to the two-step oxidation and photolysis resulting in the exclusive formation of the homodimeric products $\mathbf{1 7}$ 
and 28 (55\% and 51\% yield, respectively). HPLC analysis of the crude reaction mixture did not reveal any of the heterodimeric product 29, highlighting the superb level of control in this guided fragment union via solvent-caged radical recombination. ${ }^{23]}$ Further investigation into the solventcage effects revealed high selectivity for recombination versus disproportionation. Oxidation and subsequent photolysis of sulfamide (+)-20 (Scheme 10) in the presence of excess 1,4-cyclohexadiene (5 equiv), a $\mathrm{H}$-atom donor, resulted in an almost equal mixture of the dimeric product (+)-17 (45\% yield) and the corresponding $\mathrm{C}(3 \mathrm{a})-\mathrm{H}$ reduction product $(+)-\mathbf{3 0}(22 \%$ yield, $\sim 1: 1$ molar ratio). Increasing the amount of 1,4-cyclohexadiene (20 equiv) afforded a similar result. ${ }^{[23]}$ Comparatively, treatment of tricyclic bromide (+)-15 (Scheme 11) with tris(triphenylphosphine) cobalt(I) chloride in the presence of 1,4-cyclohexadiene ( 5 or 20 equiv) afforded exclusively the reduction product $(+)-30$ in $54 \%$ and $82 \%$ yields, respectively. These results are consistent with a solvent-cagedirected radical-pair combination in our diazene-based directed fragment assembly.

This newly developed directed fragment assembly holds great potential for application in complex settings as illustrated by successful preparation of heterodimeric products illustrated in Fig. 1. ${ }^{[23]}$ Notably, heterodimer (+)-31 resulting from the coupling of a tetracyclic diketopiperazine with a cyclotryptamine derivative represents a directed and exclusive heterodimeric union of dissimilar subunits. Furthermore, selective synthesis of meso-16, a structural core found in many cyclotryptamine natural products, can be directly compared to our exploratory and unguided synthesis utilizing our cobalt promoted cyclotryptamine dimerization (Scheme 5). Additionally, aryl-alkyl diazenes were also found to undergo fragmentation and coupling to afford C(3a)-arylated products (+)-32 and (+)-33, exhibiting a $\mathrm{C}_{\mathrm{sp} 3}-\mathrm{C}_{\mathrm{sp} 2}$ linkage found in a variety of natural alkaloids, with similar efficiency to the corresponding alkyl- alkyl diazenes. ${ }^{[23]}$ Overall the application of diazenes as traceless linkers and radical precursors allows for the generation of localized radicals whose union within the solvent-cage provides an exciting general strategy for directed heterodimeric assembly.

In conclusion, the synthesis of a variety of dimeric alkaloids has been realized with the aid of selective radical coupling and oxidation reactions. Furthermore heterodimeric molecular substructures have been accessed via diazene fragmentation followed by solvent-caged radical recombination. These newly developed general strategies allow for the selective synthesis of a variety of cyclotryptamine and cyclotryptophan-based natural products.

\section{Acknowledgements}

We acknowledge financial support by NIHNIGMS (GM089732) and Amgen. M.M. is a Camille Dreyfus Teacher-Scholar.

Received: March 18, 2012

[1] a) L. Yet, Tetrahedron 1999, 55, 9349; b) K. C. Majumdar, P. K. Basu, P. P. Mukhopadhyay, Tetrahedron 2004, 60, 6239.

[2] I. Denissova, L. Barriault, Tetrahedron 2003, 59, 10105.

[3] a) G. J. Rowlands, Tetrahedron 2009, 65, 8603; b) G. J. Rowlands, Tetrahedron 2010, 66, 1593.

[4] J. Kim, J. A. Ashenhurst, M. Movassaghi, Science 2009, 324, 238.

[5] a) R. B. Woodward, N. C. Yang, T. J. Katz, V. M. Clark, J. Harley-Mason, R. F. J. Ingleby, N. Sheppard, Proc. Chem. Soc. 1960, 76; b) R. Robinson, H. J. Teuber, Chem. Ind. 1954, 783.

[6] a) J. B. Hendrickson, R. Rees, R. Göschke, Proc Chem. Soc. 1962, 383; b) T. Hino, S. Yamada, Tetrahedron Lett. 1963, 4, 1757; c) A. I. Scott, F. McCapra, E. S. Hall, J. Am. Chem. Soc. 1964, 86, 302; d) M. Nakagawa, H. Sugumi, S. Kodato, T. Hino, Tetrahedron Lett. 1991, 22, 5323; e) C.-L. Fang, S. Horne, N. Taylor, R. Rodrigo, J. Am. Chem. Soc. 1994, 116, 9480; f) M. Somei, N. Osikiri, M. Hasegawa, F. Yamada, Heterocycles 1999, 51, 1237; g) H. Ishikawa, H. Takayama, N. Aimi, Tetrahedron Lett. 2002, 43, 5637; h) T. Matsuda, M. Kitajima, H. Takayama, Heterocycles 2005, 65, 1031.

[7] M. A. Schmidt, M. Movassaghi, Synlett 2008, 313.

[8] M. Movassaghi, M. A. Schmidt, Angew. Chem. Int. Ed. 2007, 46, 3725 .

[9] For seminal reports, see: a) M. Bruncko, D. Crich, R. Samy, J. Org. Chem. 1994, 59, 5543; b) S. P. Marsden, K. M. Depew, S. J. Danishefsky, J. Am. Chem. Soc. 1994, 116, 11143; c) K. M. Depew, S. P. Marsden, D. Zatorska, A. Zatorski, W. G. Bornmann, S. J. Danishefsky, J. Am. Chem. Soc. 1999, 121, 11953.

[10] For reviews of hexahydropyrroloindoles, see: a) D. Crich, A. Banerjee, Acc. Chem. Res. 2007, 40, 151; b) P. Ruiz-Sanchis, S. A. Savina, F. Albericio, M. Álvarez, Chem. Eur. J. 2011, 17, 1388.

[11] a) M. Movassaghi, M. A. Schmidt, J. A Ashenhurst, Angew. Chem. Int. Ed. 2008, 47, 1485; b) J. Kim, M. Movassaghi, J. Am. Chem. Soc. 2010, 132, 14376.

[12] For other applications of our cobalt-promoted chemistry in the synthesis of cyclotryptaminebased alkaloids, see: a) C. Pérez-Balado, Á. R. de Lera, Org. Lett. 2008, 10, 3701; b) C. Pérez- 
Balado, P. Rodríguez-Grãna, Á. R. de Lera, Chem.-Eur. J. 2009, 15, 9928; c) E. Iwasa, Y Hamashima, S. Fujishiro, E. Higuchi, A. Ito, M. Yoshida, M. Sodeoka, J. Am. Chem. Soc. 2010 132, 4078; d) K. Foo, T. Newhouse, I. Mori, H. Takayama, P. S. Baran, Angew. Chem., Int. Ed. 2011, 50, 2716.

[13] J. Kim, M. Movassaghi, Chem. Soc. Rev. 2009, 38,3035 .

[14] M. Movassaghi, D. S. Siegel, S. Han, Chem. Sci. 2010, 1, 561.

[15] This hypothesis was consistent with earlier feeding experiments using labeled cysteine; see: a) N. R. Towers, D. E. Wright, N. Z. J. Agric. Res. 1969, 12, 275; b) G. W. Kirby, D. J. Robins, in 'The Biosynthesis of Mycotoxins: A Study in Secondary Metabolism', Ed. P. S. Steyn, Academic Press, New York, 1980, p. 301

[16] This hypothesis diverged from an earlier hypothesis involving N-hydroxylation; see: J. D.
M. Herscheid, R. J. F. Nivard, M. W. Tijhuis, H. C. J. Ottenheijm, J. Org. Chem. 1980, 45, 1885.

[17] For the influence of proximal inductively withdrawing substituents on the formation of $\mathrm{N}$-acyliminium ions, see ref. [11b] and for an application of this bromination method to a system possessing vicinal stabilizing groups, see ref. [12c].

[18] K. A. Gardner, J. M. Mayer, Science 1995, 269, 1849

[19] T. Sala, M. V. Sargent, J. Chem. Soc. Chem. Commun. 1978, 253.

[20] T. Strassner, K. N. Houk, J. Am. Chem. Soc. 2000, 122, 7821.

[21] R. G. Eccles, Proc. Am. Pharm. Assoc. 1888, $84,382$.

[22] C. Takahashi, K, Minoura, T. Takeshi, A. Numata, K. Kushida, T. Shingu, S. Hagishita, H. Nakai, T. Sato, H. Harada, Tetrahedron 1995, 51,3483 .
[23] M. Movassaghi, O. K. Ahamad, S. P. Lathrop, J. Am. Chem. Soc. 2011, 133, 13002.

[24] a) E. M. Kosower, Acc. Chem. Res. 1971, 4 193; b) T. Tsuji, E. M. Kosower, J. Am. Chem. Soc. 1971, 93, 1992; c) P. S. Engel, Acc. Chem. Res. 1973, 6, 275; d) P. S. Engel, Chem. Rev. 1980, 80,99

[25] a) R. D. Little, Chem. Rev. 1996, 96, 93; b) V. Mascitti, E. J. Corey, J. Am. Chem. Soc. 2004, 126,15664 ; c) P. A. Wender, J.-M. Kee, J. M. Washington, Science 2008, 320, 649.

[26] a) S. F. Nelsen, P. D. Bartlett, J. Am. Chem. Soc 1966, 88,137 ; b) S. F. Nelsen, P. D. Bartlett, $J$. Am. Chem. Soc. 1966, 88, 143; c) F. D. Greene, M. A. Berwick, J. C. Stowell, J. Am. Chem. Soc. 1970, 92, 867; d) N. A. Porter, L. J. Marnett, J. Am. Chem. Soc. 1973, 95, 4361; e) R. C. Neuman Jr., R. H. Grow, G. A. Binegar, H. J. Gunderson, J. Org. Chem. 1990, 55, 2682; f) P. S. Engel, L. Pan, T. Ying, L. B. Alemany, J. Am. Chem. Soc. 2001, 123, 3706. 